\title{
Levantamento do conhecimento da população urbana de Inconfidentes/MG sobre orgânicos
}

Sindynara Ferreira ${ }^{1}$

José Annes Marinho²

\section{Resumo}

Os sistemas de produção agrícola envolvem processos sociais. A agricultura orgânica tem como princípio produzir alimentos sem defensivos agrícolas e favorecer a interação do homem com os recursos naturais de modo a preservar esses últimos para as gerações futuras. Inconfidentes fica no Sul de Minas Gerais e destaca-se como produtor de alimentos comercializados in natura na própria região. $O$ objetivo deste estudo foi auferir o conhecimento da população local a respeito da agricultura orgânica em relação à convencional. Verificou-se que é necessário divulgar essas definições e inserir os orgânicos em supermercados e quitandas. Há demanda de informações sobre o que é agricultura orgânica e o que a distingue da agricultura convencional. A satisfação dessa demanda seria facilitada se os produtores de orgânicos da região cobrassem mais marketing local das certificadoras. Ações de educação ambiental também poderiam auxiliar a expansão do mercado de orgânicos em Inconfidentes. Palavras-chave: Agricultura orgânica. Agricultura convencional. Comércio. Benefícios.

\section{Introdução}

O Sul de Minas Gerais destaca-se como importante polo de produção agrícola, sendo que muito dessa produção é comercializado de forma in natura na própria região. A sociedade atual demanda não somente uma grande quantidade de alimentos, como também que os mesmos tenham determinadas características (Moura et al., 2010).

A prática de produção tem normalmente sido desenvolvida por produtores especializados, que usam tecnologia convencional de produção, utilizam intensamente insumos químicos, cobrem o solo com plástico e plantam sucessivamente em uma mesma área. Essas práticas convencionais têm acarretado problemas na conservação do solo e da água e no manejo do ambiente como um todo. Paralelamente, os consumidores a cada ano se interessam mais por alimentos saudáveis, e então os produtos orgânicos têm se mostrado como alternativa de consumo.

A agricultura orgânica tem por princípio estabelecer sistemas de produção com base em tecnologias de processos, ou seja, um conjunto de procedimentos que envolvem a planta, o solo e as condições climáticas e que produzem um alimento sadio, com características e sabores que atendam às expectativas do consumidor (PENTEADO, 2000). A agricultura orgânica tem surgido como alternativa para solucionar os problemas da agricultura convencional, cujo objetivo prioritário tem sido maximizar a produção (DAROLT, 2004). Para que sejam considerados orgânicos, estes produtos

1 Instituto Federal de Educação, Ciência e Tecnologia do Sul de Minas Gerais, campus Inconfidentes - Professora Efetiva - Inconfidentes - Minas Gerais - Brasil - sindynara.ferreira@ifs.ifsuldeminas.edu.br - (35) 3464-1200 - Praça Tiradentes, 416, Centro, 37.576000, Inconfidentes/MG.

2 Associação Nacional de Defesa Vegetal, ANDEF/SP - Gerente de Educação - São Paulo - São Paulo - Brasil - annes@andef.com. br - (11) 3087-5037 - Rua Capitão Antonio Rosa, 376, $13^{\circ}$ andar, 01.443-10, São Paulo/SP. 
precisam receber um selo de certificação que identifica a procedência do produto e as práticas de cultivo (que devem ser livres de fertilizantes sintéticos ou defensivos). Os certificados também atestam as condições de trabalho e remuneração das pessoas envolvidas na produção. Estes certificados são emitidos por agências certificadoras, responsáveis, ainda, pela inspeção e pela orientação do processo produtivo destes produtos (DENOBILE, 2005).

A agricultura orgânica é uma oportunidade para o produtor, pois ele pode ampliar vendas e receitas ao atender a um segmento crescente do mercado enquanto continua a desenvolver a mesma atividade, porém de uma forma que Ihe trará, no médio e no longo prazo, melhores condições de manejo de sua propriedade e de preservação dos seus recursos naturais.

Darolt (2003) e Karan e Zoldan (2003) mostraram que o mercado de consumo da agricultura orgânica se expande em âmbito nacional e internacional. Essa expansão está associada ao aumento do custo dos produtos convencionais, à degradação do meio ambiente e à crescente exigência dos consumidores por produtos isentos de substâncias químicas ou geneticamente modificados.

Compreender o processo de produção é importante para o consumir porque aumenta seu poder de escolha/preferência. Daí a relevância deste trabalho, cujo objetivo foi levantar o conhecimento da população local do município de Inconfidentes sobre produtos orgânicos.

\section{Materiais e métodos}

Optamos por uma pesquisa qualitativa. Essa modalidade de pesquisa não enumera nem mede eventos através de instrumental estatístico de análise de dados. A pesquisa qualitativa obtém dados descritivos através do contato direto e interativo do pesquisador com o objeto de estudo (NEVES, 1996).

Nossa área de estudo foi o município de Inconfidentes, o qual se localiza a 869 metros de altitude, com uma posição marcada pelas coordenadas geográficas de $22^{\circ} 19^{\prime} 00^{\prime \prime}$ de altitude e $46^{\circ}$ 19' 40" de longitude. Está localizada ao Sul do Estado de Minas Gerais e se assenta numa área de 145 quilômetros quadrados (PREFEITURA MUNICIPAL DE INCONFIDENTES, 2011). Segundo informações do funcionário Francisco Volney Costa em 03/08/2012, o município conta com 32 bairros, dos quais 28 estão na zona rural e 4 estão na zona urbana, entretanto a a população urbana supera a rural. Os bairros na zona urbana são: Centro, Vila Nossa Senhora Aparecida, Santa Luzia e Santa Izabel. Os bairros da zona rural são: Alto da Boa Ventura, Alto do Mogi, Angu Frio, Boa Ventura, Boa Vista da Adelaide, Boa Vista dos Góes, Boa Vista dos Freitas, Boa Vista das Pitangueiras, Bom Jardim, Borges, Cambuizinho, Córrego Grande, Córrego da Onça, Córrego do Urutu, Escritório Velho, Grama, Monjolinho, Morro Alto, Paredes, Pavoeiro, Pessegueiro, Pinhalzinho dos Gois, Pitanga, Porantava ("Cavaco"), Posses, Romas, Santa Isabel, Soledade do Mogi.

Inconfidentes conta atualmente com 6.859 habitantes (INSTITUTO BRASILEIRO DE GEOGRAFIA E ESTATÍSTICA, 2011). Delimitamos como objeto de estudo somente a zona urbana e estipulamos contatar aproximadamente $4 \%$ do total de habitantes (cerca de 272 entrevistados).

A coleta de dados ocorreu durante o mês de dezembro de 2011 . Foi realizada por meio de entrevista com auxilio de um questionário. O questionário teve perguntas padronizadas, o que facilitou a tabulação das respostas. As perguntas eram algumas fechadas e outras abertas, de escolha única e múltipla, e abordavam o conhecimento sobre produtos orgânicos em áreas como: produção e comercialização de orgânicos no município; diferença de produção: orgânicos versus convencional; selos que garantem que o produto é orgânico; preferências para o consumo; e receitas orgânicas. 


\section{Resultados e discussão}

Dos 272 entrevistados, tivemos 140 pessoas do sexo feminino e 132 pessoas do sexo masculino, uma relação que podemos considerar parcialmente homogênea no ambiente amostral. 25,73\% de todos os entrevistados estavam na faixa etária dos 21 aos 30 anos (Figura 1) faixa esta que tende a acompanhar as novas tecnologias e meios de divulgação de informações.

Figura 1. Faixa etária dos entrevistados da zona urbana de Inconfidentes/MG. Inconfidentes/MG, 2013.

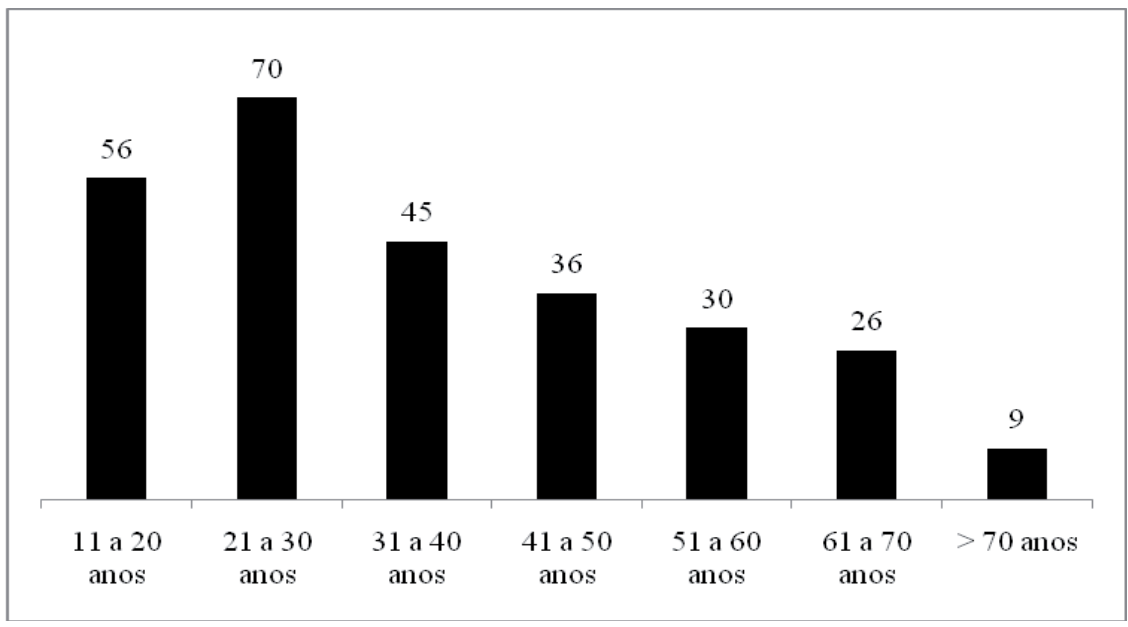

Fonte: Elaboração própria.

$47,42 \%$ dos entrevistados concluíram o ensino médio, 25\% concluíram o ensino fundamental, $23,16 \%$ completaram o ensino superior e apenas $4,42 \%$ possuíam pós-graduação.

Inconfidentes é tido como a "Capital Nacional do Crochê" porque possui mais de sessenta malharias.

$\mathrm{Na}$ zona rural, contudo, predominam pequenas e médias propriedades dedicadas ao cultivo da bucha, do café, das olerícolas como a batata e o alho; e à criação de gado de leite e de corte.

A maioria dos entrevistados $(51,47 \%)$ tem renda entre 1 e 3 salários (renda que pode ser considerada média para a região). 27,57\% tem renda mensal familiar de um salário mínimo; 15,08\% de quatro a seis salários; $3.31 \%$ de sete a nove salários; $1,10 \%$ de dez a doze salários; e 1,47\% recebem mais que doze salários.

Quanto aos critérios de compra de produtos agrícolas, $61,76 \%$ dos entrevistados atentam para a característica de firmeza; $36,02 \%$ atentam para a característica de cor; $34.92 \%$ atentam para o cheiro; $25.73 \%$ consideram o tamanho; e $18.38 \%$ consideram outras características (como validade, preço, depende do produto e necessidade).

Estudos conduzidos por Karam (2002), Karam e Zoldan (2003) e Darolt (2003) indicaram que os consumidores de produtos orgânicos consideram que as características intrínsecas relacionadas à composição destes produtos são mais importantes do que a aparência e o sabor deles. Em nenhum momento da pesquisa foi relatado pelos entrevistados que a beleza dos produtos influenciasse 0 ato da compra. É importante ressaltar que pesquisas já revelaram que os consumidores não acreditam que a aparência externa permite diferenciar um produto orgânico de um convencional (MONTEIRO et al., 2003).

Os entrevistados foram questionados sobre a frequência com que costumavam ler os rótulos dos produtos e se, após a compra, usavam todo o produto ou deixavam sobras. 
Somente $38 \%$ lê rotineiramente os rótulos no ato da compra, 37\% lê quando acha pertinente e $25 \%$ não lê os rótulos.

Quanto ao uso do produto após a aquisição, 54.37\% dos entrevistados utilizam o produto por completo e $45.63 \%$ deixam ocorrer sobras dos produtos.

Do total de entrevistados, $84,55 \%$ já ouviu falar em agricultura orgânica e metade dos entrevistados, ou seja, $50 \%$, relatou que o meio de divulgação por meio do qual conseguiram informação sobre produtos orgânicos era a televisão (TV), seguido pela escola, com 29,77\% de relatos (Figura 2).

Figura 2. Meio de comunicação privilegiado para saber sobre produtos orgânicos. Inconfidentes/MG, 2013.

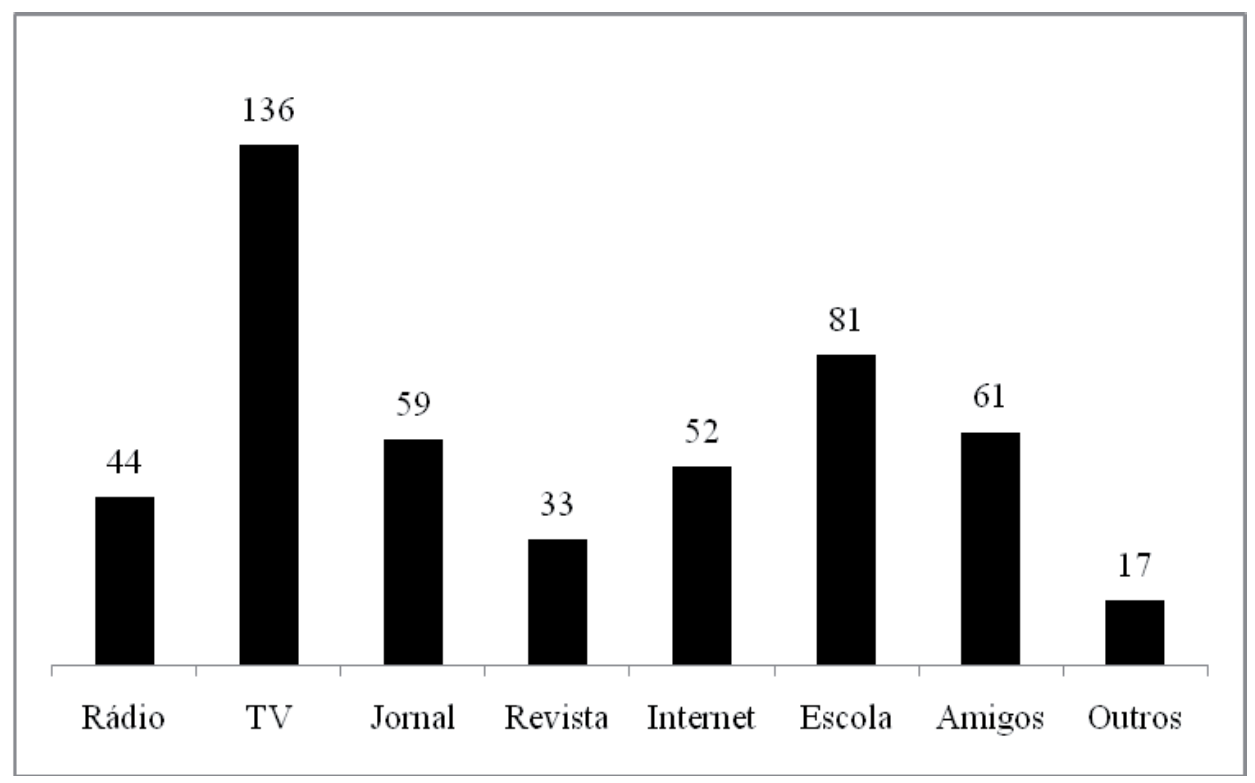

Fonte: Elaboração própria.

Esses dados demonstram o poder informativo da televisão e corroboram os dados encontrados também por Casemiro e Trevizan (2009). Porém estes resultados se contrapõem aos encontrados por Moura et al. (2010), segundo os quais a comunicação acerca dos produtos orgânicos é deficiente, com poucas reportagens que divulgam seus benefícios, as diferenças entre produtos orgânicos e convencionais, e o processo de distribuição para o consumidor.

Em outra questão, foi levantado se os entrevistados conseguiam diferenciar agricultura orgânica da agricultura convencional. 50,73\% respondeu que sabia diferenciar e somente $30,88 \%$ afirmou conhecer selos que garantem que o produto é orgânico.

Para um produto ser conhecido, é necessário utilizar estratégias de comunicação, propaganda, publicidade, relações públicas, promoção de vendas e merchandising. Conforme afirma Pinheiro e Gulo (2005, p. 23), "o processo de comunicação com o mercado visa criar imagem e apoiar as vendas do produto, informando sobre sua existência, levando conhecimento sobre seu benefício".

35,66\% dos entrevistados conhecem estabelecimentos que comercializam produtos orgânicos, e citaram o Supermercado Bonamigo, a Cooperativa Escola, a Quitanda do Vadinho e a Quitanda da Gislene. Averiguamos esses estabelecimentos em janeiro de 2012 e constatamos que nenhum comercializava produtos orgânicos. 50,36\% dos entrevistados acreditam que os produtos orgânicos duram mais do que os produtos convencionais.

$72.62 \%$ dos entrevistados já consumiram algum tipo de produto orgânico, enquanto $27.38 \%$ dos entrevistados ainda não tiveram oportunidade de consumir produtos orgânicos. 
$57 \%$ dos entrevistados responderam que consomem produtos orgânicos porque esses produtos trazem benefícios à saúde; $23 \%$ por causa do sabor; $14 \%$ porque se preocupam com o meio ambiente; $6 \%$ porque conhecem alguém que consome produtos orgânicos; e $1 \%$ por causa de alergia a outros produtos.

Figura 3. Motivos para consumir produtos orgânicos. Inconfidentes/MG, 2013.

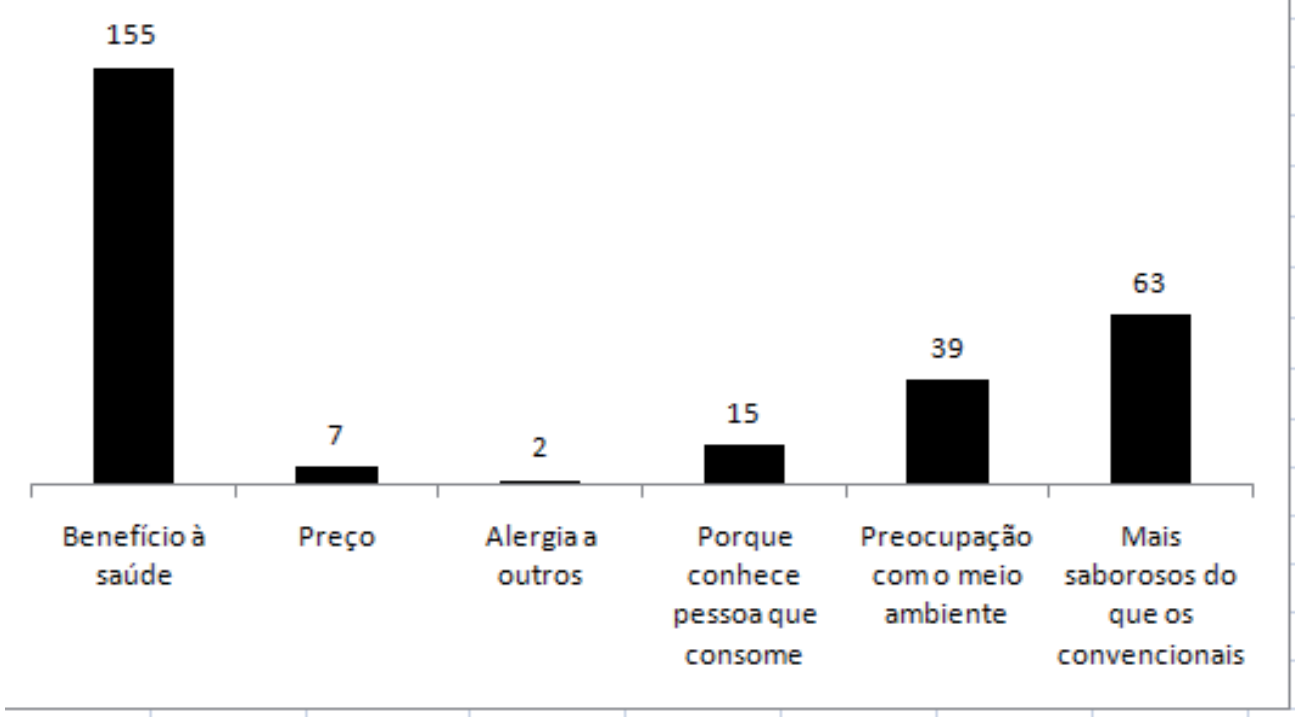

Fonte: Elaboração própria.

Estas respostas vão ao encontro dos trabalhos de Moura et al. (2010), Vilas Boas, Sette e Pimenta (2008) e Monteiro et al. (2003), de acordo com os quais os consumidores de produtos orgânicos consideraram que esses são mais saudáveis do que os convencionais, possuem sabor mais agradável e ajudam a preservar o meio-ambiente.

$69.85 \%$ dos entrevistados afirmaram que os produtos orgânicos custam mais caro do que os produtos convencionais. Moura et al. (2010) também apontou que o preço elevado se destaque dentre as causas do baixo consumo dos orgânicos. Em algumas situações relatadas, a diferença entre os produtos orgânicos e convencionais chegou a mais de $400 \%$. A maioria dos entrevistados $(29,41 \%)$ explicou os preços elevados como consequência da produção em pequena escala (Figura 4). 
Figura 4. Motivos dos produtos orgânicos possuírem preços mais elevados que os produtos convencionais. Inconfidentes/MG, 2013.

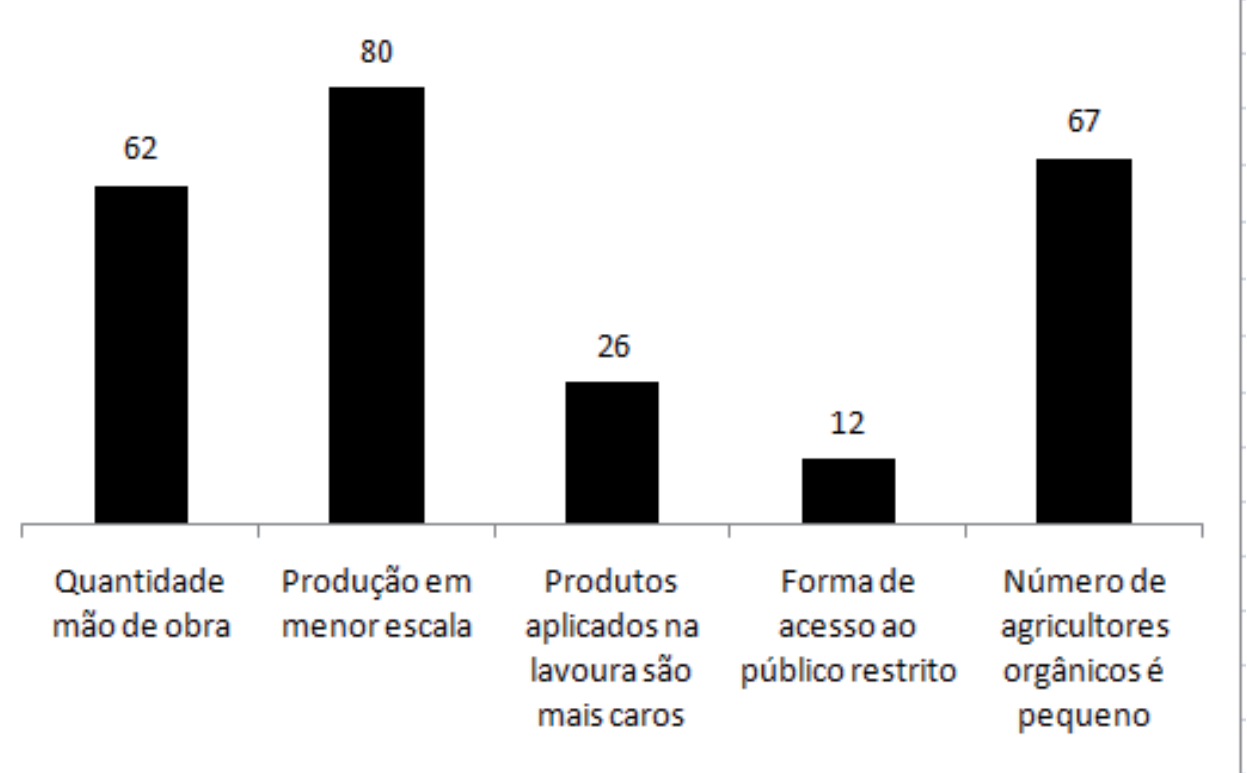

Fonte: Elaboração própria.

Os entrevistados foram questionados sobre o fator que mais dificulta o acesso a produtos orgânicos no município. $43,75 \%$ relatou que não encontra o produto no mercado, fator seguido de pouca variedade, preço, aspecto visual, outros e qualidade insatisfatória (Figura 5).

Figura 5. Resultado da entrevista sobre fator que dificulta o acesso a produtos orgânicos no município. Inconfidentes/MG, 2013.

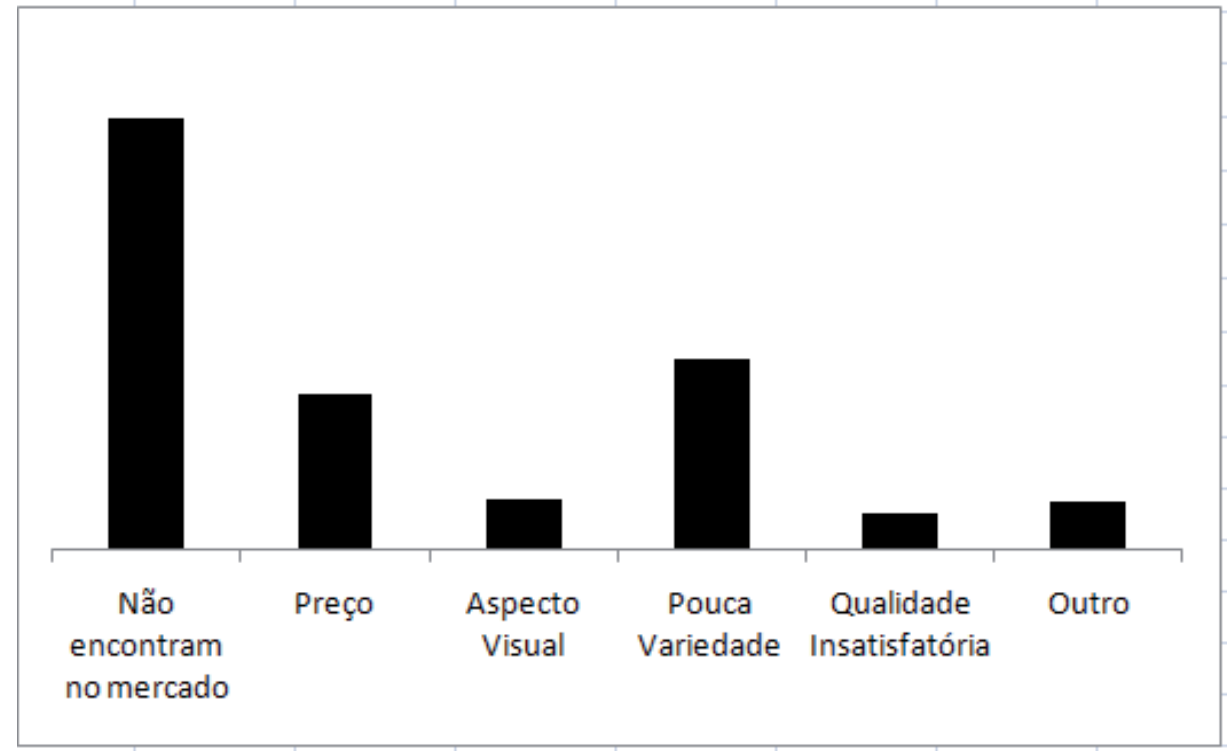

Fonte: Elaboração própria.

70.59\% dos entrevistados responderam que não possuem receitas de comidas com produtos orgânicos.

Em pesquisas sobre o acesso aos produtos orgânicos, Monteiro et al. (2003) apontaram que , 47\% dos entrevistados acharam difícil o acesso a produtos orgânicos. Moura et al. (2010) relata- 
ram alguns problemas enfrentados pelos consumidores de produtos orgânicos e destacaram o fator distribuição. Apesar de começar a ganhar espaço nas prateleiras de supermercados, os respondentes consideraram a sua distribuição ainda muito limitada. Para comprar produtos orgânicos, na maioria das vezes os consumidores têm de ir às feiras ou lojas especializadas. Além disso, a variedade e a oferta de produtos orgânicos nas grandes redes de supermercados foram consideradas ainda insatisfatórias pelos entrevistados.

\section{Conclusões}

O estudo realizado mostra que a informação sobre alimentos orgânicos começa a fazer parte do senso comum das pessoas.

Verifica-se a necessidade de divulgar os produtos orgânicos em Inconfidentes e de disponibilizá-los no comércio local.

\section{Survey of knowledge of urban population in Inconfidentes/MG on organic agriculture}

\section{Abstract}

The agricultural production systems involve social processes. Organic agriculture has as a principle the production of food without pesticides and encouraging the interaction of human and natural resources to preserve the latest for future generations. Inconfidentes is in southern Minas Gerais and stands out as a producer of fresh foods marketed in the region itself. The aim of this study was to derive the knowledge of local people about organic farming compared to conventional one. It was found that it is necessary to disclose these settings and enter the organic supermarkets and greengrocers. There is a demand for information about what is organic farming and what distinguishes it from conventional agriculture. The satisfaction of this demand would be facilitated if the producers of the region requested more local marketing of certification. Environmental education could also help to expand the market for organic in Inconfidentes.

Keywords: Organic Agriculture. Conventional agriculture. Commerce. Benefits;

\section{Agradecimentos}

Aos alunos/colegas que me ajudaram sem mensurar as dificuldades durante a pesquisa: Aline Cristina Mendes, Cristiano de Castro Leite, Danilo Cândido da Silva, Guilherme Giannini Loyola, Jessica Kauane Ferreira Passos, Jislaine Samara Mendes, João Marcos Carvalho Vasconcelos, Marcos Robson Silva, Natália Machado Ferreira, Olivânia Ribeiro de Almeida, Rodrigo Félix Simões, Valéria Cristina Gonçalves Marins e aos colegas de trabalho Éder Clementino dos Santos e Wilson Roberto Pereira pelo auxílio na elaboração do questionário. 


\section{Referências bibliográficas}

CASEMIRO, A. D.; TREVIZAN, S. D. P. Alimentos orgânicos: desafios para o domínio público de um conceito. In: INTERNATIONAL WORKSHOP ADVANCES IN CLEANER PRODUCTION. 2., 2009, São Paulo. Proceedings... [s.I]: [s.n.], 2009. Disponível em: <http://www. advancesincleanerproduction.net/second/files/sessoes/6a/1/A.\%20D.\%20Casemiro\%20-\%20 Resumo\%20Exp.pdf>. Acesso em: 20 mai. 2013.

DAROLT, M. R. Agricultura orgânica: um estudo de sustentabilidade na região metropolitana de Curitiba, Paraná. 2004. [?] p. Tese (Doutorado). Universidade Federal do Paraná, [s.I.].

DAROLT, M. R. 0 consumidor e o mercado de produtos orgânicos. In: SIMPÓSIO A PESQUISA EM AGRICULTURA. 1., 2003, Lavras. Anais... Lavras: UFLA, 2003.

DENOBILE, T. Modelo de gestão estratégica com foco no cliente para comercialização de produtos orgânicos. 2005. 152 p. Dissertação (Mestrado). Universidade Estadual de Campinas, Campinas.

INSTITUTO BRASILEIRO DE GEOGRAFIA E ESTATÍSTICA. Censo 2010. Disponível em: <http:// www.ibge.gov.br/home/estatistica/populacao/censo2010/resultados_dou/MG2010.pdf >. Acesso em: 20 dez. 2011.

KARAN, K. F.; ZOLDAN, P. Comercialização e consumo de produtos agroecológicos: pesquisa dos locais de venda, pesquisa do consumidor região da grande. Florianópolis: Instituto Cepa/SC, 2003.

MONTEIRO, M. N de C.; SALGUEIRO, M.; COSTA, R. T. da; G. R. B. Os alimentos orgânicos e a percepção de seus atributos por parte dos consumidores. In: SEMEAD. 7., 2003. Anais... São Paulo: FEA-USP, 2003. Disponível em: < http://www.ead.fea.usp.br/Semead/7semead/paginas/ artigos\%20recebidos/marketing/MKT08_-_Os_alimentos_organicos_consumidores.PDF > . Acesso em: 23 dez. 2011.

MOURA, L. R. C. et al. Um estudo sobre o comportamento dos consumidores de produtos orgânicos. In: SEMEAD. 8., 2003. São Paulo: FEA-USP, 2003. Disponível em:<http://www.ead. fea.usp.br/semead/13semead/resultado/trabalhosPDF/579.pdf>. Acesso em: 20 mai. 2013.

NEVES, J. L. Pesquisa qualitativa: características, usos e possibilidades. Caderno de Pesquisas em Administração, São Paulo, v. 1, n. 3, 1996. Disponível em: <http://www.ead.fea.usp.br/cad-pesq/ arquivos/c03-art06.pdf>. Acesso em: 20 mai. 2013.

PENTEADO, S. R. Introdução à agricultura orgânica: normas e técnicas de cultivo. Campinas: Editora Grafimagem, 2000.

PINHEIRO, D.; GULO, J. Comunicação integrada de marketing: gestão de elementos de comunicação. São Paulo, Atlas, 2005.

PREFEITURA MUNICIPAL DE INCONFIDENTES. Dados históricos do município. Disponível em: <http://www.inconfidentes.mg.gov.br/cidade.php?codigo=2 >. Acesso 23 dez. 2011.

VILAS BOAS, L. H. de B.; SETTE, R. de S.; PIMENTA, M. L. Comportamento do consumidor de alimentos orgânicos na cidade de Uberlândia: uma aplicação da técnica Ladderning. In: CONGRESSO DA SOCIEDADE BRASILEIRA DE ECONOMIA, ADMINISTRAÇÃO E SOCIOLOGIA 
RURAL. 46., Rio Branco, 2008. Anais... Rio Branco: Sociedade Brasileira de Economia, Administração e Sociologia Rural, 2008. Disponível em: <http://www.sober.org.br/palestra/9/730. pdf > . Acesso $10 \mathrm{dez} .2011$.

\section{Histórico editorial}

Recebido: 13/02/2013

Avaliação e copidesque: 21/02/2013 a 18/06/2013 


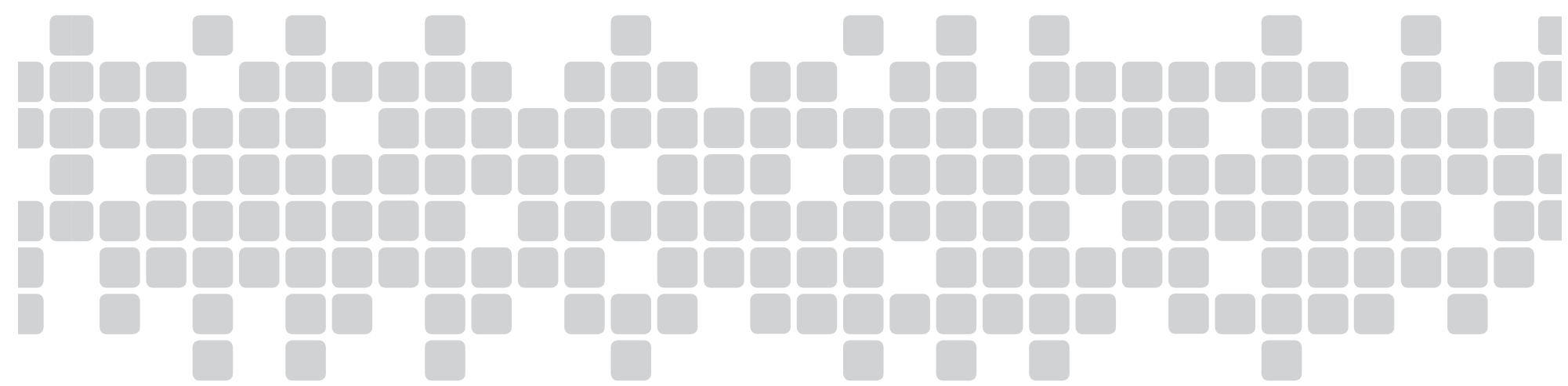

